Vort i cal, tor oi dal and compressi bl e mot i ons i n 3D MHD si mil at i ons of LHD

\begin{tabular}{|c|c|}
\hline $\begin{array}{l}\text { j our nal or } \\
\text { publ i cat i on } \mathrm{titl} \text { e }\end{array}$ & J our nal of Pl asma Physi cs \\
\hline vol une & Vol . 72 \\
\hline number & I ssue 6 \\
\hline page $r$ ange & pp. 1095- 1099 \\
\hline year & 2006-12-01 \\
\hline URL & ht t p: //hdl . handl e. net /10655/3892 \\
\hline
\end{tabular}




\title{
Vortical, toroidal and compressible motions in 3D MHD simulations of LHD
}

\author{
H. M I URA $A^{1,3}$, N. NAKA J I M A $A^{1,3}$, T. HA Y A S H I I,3 \\ and M. O K A M O T O ${ }^{1,2}$ \\ ${ }^{1}$ Theory and Computer Simulation Center, \\ National Institute for Fusion Science, 322-6 Oroshi, Toki, Gifu 509-5292, Japan \\ ${ }^{2}$ Division of Theory and Data Analysis, \\ National Institute for Fusion Science, 322-6 Oroshi, Toki, Gifu 509-5292, Japan \\ ${ }^{3}$ Department of Fusion Science, The Graduate University for Advanced Studies \\ (SOKENDAI),
}

322-6 Oroshi, Toki, Gifu 509-5292, Japan

(Received 25 August 2005 and accepted 12 June 2006)

\begin{abstract}
Direct numerical simulation of fully three-dimensional, compressible and nonlinear magnetohydrodynamic equations in the Large Helical Device is carried out in combination with the passive particle simulation. In the simulation, strong vortical motions are excited by the pressure-driven instability and form the mushroom-like structures of pressure. It is shown by the passive particles analysis that the fluid volumes around the resonant magnetic surfaces experience finite compressibility and toroidal deformation, which are both excited by the strong vortical motions. The passive particles simulation helps us to investigate local structures even for low Fourier wavenumber modes.
\end{abstract}

\section{Introduction}

The Large Helical Device (LHD) is the world's largest heliotron/torsatron type helical device. It has a set of $L=2 / M=10$ helical coils with a major radius of $3.9 \mathrm{~m}$ (Motojima et al. 1999). Recent LHD experiments revealed that plasmas can be confined relatively well even under the magnetic configuration with vacuum magnetic axis position $R_{\mathrm{ax}}=3.6 \mathrm{~m}$, which is considered unstable in the sense of Magnetohydrodynamic (MHD) instability. In order to understand how plasma survives MHD instabilities, direct numerical simulation (DNS) of fully three-dimensional, compressible and nonlinear MHD equations is carried out by our numerical code, the MHD In Non-Orthogonal System (MINOS). Details of the MINOS code and conditions of the DNS are reported in Miura et al. (2004, 2007).

In traditional linear analysis and reduced MHD simulations, toroidal flow and compressibility are often discarded. This is partly because the fluid remains incompressible if the perturbation is initially incompressible in the framework of the reduced MHD equations. The toroidal velocity can also be omitted if the perturbation is incompressible. However, we have shown that the toroidal component of the velocity grows rapidly from a weakly perturbed state. (Miura et al. 2004, 2007). 


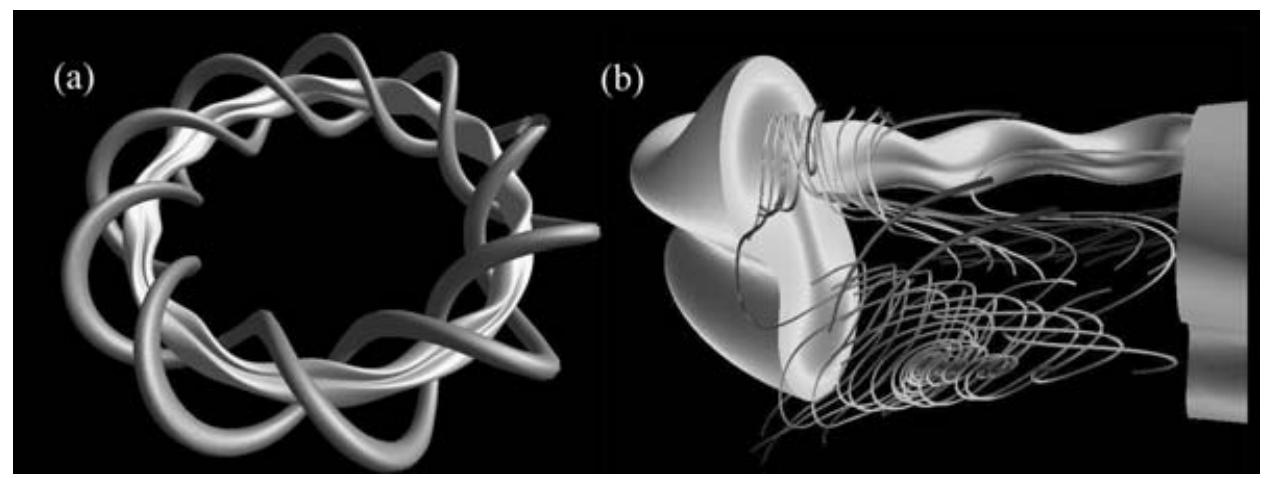

Figure 1. Full 3D views of MHD instabilities obtained by DNS. (a) Pressure isosurface with two helical coils. (b) Stream lines, pressure isosurfaces and contours on a poloidal section.

Furthermore, the compressibility works to reduce the linear growth rate to $1 / 2-$ $1 / 5$ of the growth rate obtained under the incompressible assumption. This implies that the toroidal flow and compressibility should be included in numerical studies to study the confinement mechanism of plasmas in LHD. Although we have shown the significance of these effects, the detailed mechanism by which these effects influence the nonlinear saturations of the growth still remains unclear. Aiming to clarify the mechanism, we propose an analysis with the displacement vector by the combination with the passive scalar simulation. We show below that the new approach works well and is worth incorporating into further research on this subject.

\section{DNS with passive scalar simulations}

Our simulation starts from an unstable initial equilibrium with the vacuum magnetic axis position $3.6 \mathrm{~m}$ and $\beta_{0}=4 \%$. As is reported in Miura et al. (2004), a simulation starting from this equilibrium shows strong instability driven by the pressure gradient. In the simulations, a rapid growth of unstable Fourier modes $m / n=2 / 1$ and $1 / 1$ of the pressure are observed where $m$ and $n$ are the poloidal and toroidal wavenumber in the Boozer coordinate. A typical view of the $m / n=2 / 1$ structure of the pressure is shown in Fig. 1(a). In Fig. 1(a), the isosurface of the pressure is shown with two helical coils. We clearly observe that the isosurfaces are deformed by the growth of the $m / n=2 / 1$ Fourier mode. In Fig. 1(b), stream lines, pressure isosurfaces and contours on a poloidal section are shown. Spirals of stream represent vortical motions associated with the $m / n=2 / 1$ mode. The stream lines are inclined to the toroidal direction due to the active toroidal flows. The strong vortices, which consist of two anti-parallel vortex pairs, advect each other and form the mushroom-like structures of the pressure, as are observed in the contours of the pressure in a poloidal section.

The roles of the toroidal flow and compressibility are studied more closely with the help of the passive particles simulation. In the passive particles simulation, the position of the $i$ th particle $\boldsymbol{\xi}_{i}(t)$ is tracked by solving the equation

$$
\frac{d \boldsymbol{\xi}_{i}}{d t}=\boldsymbol{v}\left(\boldsymbol{\xi}_{i}, t\right)
$$

where $\boldsymbol{v}(\boldsymbol{\xi}, t)$ is the fluid velocity at the position $\boldsymbol{\xi}$. Since the motions of particles depend only on the fluid velocity, the vector $\delta \boldsymbol{\xi}=\boldsymbol{\xi}(t)-\boldsymbol{\xi}(t=0)$ represents the 


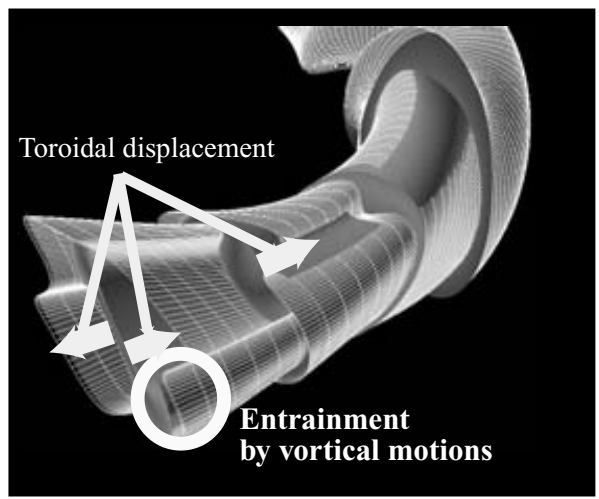

Figure 2. Deformations of passive surfaces. Poloidal cross-sections of the pressure contour and the $m / n=2 / 1$ passive surface coincide with each other at the initial time.

displacement vector which is often used to study the linear instability of torus plasmas (Friedberg 1987). The particles are initially located on the rational surfaces $\iota / 2 \pi=1 / 2,2 / 3,3 / 4,1$ uniformly on the $\theta-\zeta$ plane of the Boozer coordinate where $\theta$ and $\zeta$ are the poloidal and toroidal angles, respectively, composing the passive surfaces. We also put particles on the neighboring surfaces to each rotational surfaces for use later. The passive surfaces coincide with the resonant magnetic surfaces if the magnetic fields are frozen into the fluid, as they are in ideal MHD. Although the passive surfaces depart from the resonant magnetic surfaces because of the nonideal MHD effects, it is still worth studying the behavior of them because it serves to study the roles of physical elements such as toroidal flows and compressibility.

First, we study the deformations of the passive surfaces. In Fig. 2, the passive surfaces are shown with isosurfaces and contours on a poloidal plane of the pressure at the saturation time of the energy growth. The white meshes represent the passive surfaces. The vertexes of meshes are the position of passive particles. The inner-most surface in Fig. 2 coincides initially with the rotational surface $\iota / 2 \pi=0.5$. In the course of the time evolution, the surface is deformed into spirals by the roll-up of the vortices. It can be verified by studying the toroidal displacement $\xi \cdot \boldsymbol{B} /|\boldsymbol{B}|$, where $\boldsymbol{B}$ is the magnetic field vector, that the surface is also elongated or compressed into the toroidal direction by the toroidal flows, although it may not be clearly seen in Fig. 2. The toroidal deformation is to the left-hand side of the paper in the inner side of the torus and to the right-hand side in the outer side. The toroidal deformation is likely an $m / n=1 / 1$ structure. The observation is consistent with the analysis by means of the Fourier power spectra of the parallel velocity, reported in Miura et al. (2007). The $m / n=1 / 1$ toroidal flow is excited by the couplings of the $m / n=2 / 1$ primarily unstable mode and $m / n=1 / 0$ perturbation added to the system due to noise in the MHD equilibrium. While the directions of fluid motions and its time history are not observed from the streamlines in Fig. 1(b), the passive surfaces in Fig. 2 represent the history of the fluid motions. The passive surface representation helps us to understand the evolutions of fluid motions, especially on the rational surface, and helps us to understand the evolution of the MHD instability.

Next the significance of compressibility is investigated. Although it is possible to estimate the local compressibility by studying the dilatational field $\nabla \cdot \boldsymbol{v}$, it becomes easier by making use of the passive particles informations especially when we are 


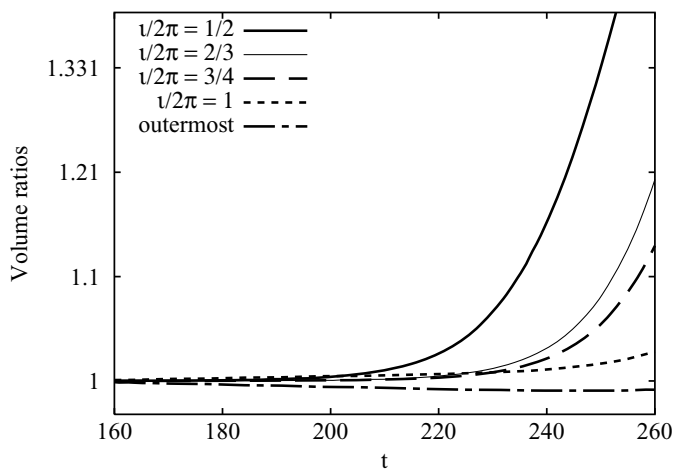

Figure 3. Ratio of the volumes of small rectangular boxes to their initial volumes.

interested in the compressibility in the neighbourhood of the rational surfaces. The six vertices of a rectangular box are composed of the passive particles neighboringeach other. Suppose that a position of one vertex is identified by the sets of variables $(\iota / 2 \pi, \theta, \zeta)$. Then the other five vertices are given by shifting the position to $\delta \iota, \delta \theta$, $\delta \zeta$ in each direction, respectively. The volumes become larger or smaller in their course of time evolutions if they experience some compressibility. In Fig. 3, time evolutions of the volumes of the small rectangular boxes on some typical rotational surfaces are shown. The time region is restricted to $160 \leqslant t / \tau_{\mathrm{A}} \leqslant 260$, that is, from the middle of the linear regime to the beginning of the nonlinear stage, so that the separation between two neighboring particles does not become too large. The volumes are all averaged over each rotational surface and normalized by the initial volume. We find in Fig. 3 that the volume of the elements which are initially located on the $\iota / 2 \pi=1 / 2$ surface grows rapidly. The volumes in the plasma cores (that is, $\iota / 2 \pi=1 / 2,2 / 3$ and $3 / 4$ surfaces) experience expansion of the volume, while the volumes in the outermost surface of the magnetic field line shrink. Note that the compressibility is weak in the simulation. The root mean square of the dilatation $\nabla \cdot \boldsymbol{v}$ is roughly $1 / 10-1 / 100$ of that of the vorticity $\nabla \times \boldsymbol{v}$. However, the analysis in the above shows that the local contributions of compressibility around the magnetic surfaces is never negligible.

We emphasize here again that all rectangular boxes distributed on the rational surfaces experience finite expansion or compression of the volume. Since the passive particles essentially represent the displacement vectors adopted in the linear MHD analysis, the large change of the volumes shows that finite compressibility works to the dynamics on the displacement vector. While the volume change could also be studied by evaluating the dilatation of the velocity field, $\nabla \cdot \boldsymbol{v}$, the passive vector information can further be used to study the parallel and perpendicular displacement and so on. The passive particle information can provide us with rich information about plasma motion.

\section{Concluding remarks}

We have studied the fluid motions associated with the growth of instability in LHD. The pressure-driven instability excites the strong $m / n=2 / 1$ vortex pairs, which in turn generates considerable strength of toroidal flows. Analysis with the passive particle simulations have revealed that the volumes which are put 
initially on rotational surfaces experience finite compressibility, even though the mean compressibility is weak. Our study in this article shows that the passive particle simulations can serve to explore various aspects of local plasma motions.

\section{References}

Motojima, O. et al. 1999 Initial physics achievements of Large Helical Device experiments. Phys. Plasmas 6, 225-245.

Miura, H. et al. 2004 Non-disruptive MHD dynamics in inward-shifted LHD configurations In: Procedings of the 19th IAEA Fusion Energy Conference, IAEA-CSP-25/CD, http://www-naweb.IAEA.org/napc/physics.fec.htm.

Miura, H. et al. 2005, to appear in Fusion Science and Technology (2007).

Freidberg, J. P. 1987 In: Ideal Magnetohydrodynamics, New York: Plenum Press. 